\title{
Factors Behind Low NPENT Rate in Bauchi State in 2015
}

\author{
Jalal-Eddeen Abubakar Saleh", *, Rui Gama Vaz ${ }^{2}$, Fiona Braka ${ }^{3}$, Khaled Abdelrahim¹, \\ Adamu Ibrahim Ningi ${ }^{1}$, Isa Mohammed Bello ${ }^{1}$
}

${ }^{1}$ EPI-IVE, World Health Organization, Bauchi Zonal Office, Bauchi State, Nigeria

${ }^{2}$ WR/NIE, World Health Organization, Abuja, Nigeria

${ }^{3}$ EPI-IVE, World Health Organization, Abuja, Nigeria

Email address:

drjalals@yahoo.com (Jalal-Eddeen A. S.)

${ }^{*}$ Corresponding author

\section{To cite this article:}

Jalal-Eddeen Abubakar Saleh, Rui Gama Vaz, Fiona Braka, Khaled Abdelrahim, Adamu Ibrahim Ningi, Isa Mohammed Bello. Factors Behind Low NPENT Rate in Bauchi State in 2015. Science Journal of Public Health. Vol. 4, No. 4, 2016, pp. $342-345$.

doi: 10.11648/j.sjph.20160404.21

Received: June 5, 2016; Accepted: June 13, 2016; Published: July 4, 2016

\begin{abstract}
The need for a country or region to achieve certification as polio-free requires spending at least 3 consecutive years without a reported case of polio. Meeting the acute flaccid paralysis (AFP) core surveillance indicators forms an important requirement during the stipulated period towards achieving certification. In addition to meeting the AFP core indicators, non-polio enteroviruses (NPENT) rate is key in evaluating whether the reported AFP meets the minimum required to be tag as true AFP. This cross-sectional quantitative study distributed questionnaires to 20 disease surveillance and notification officers (DSNOs) on the AFP surveillance network of Bauchi State, Nigeria. The responses were collated and analyzed accordingly. The result shows that although $20(100 \%)$ DSNOs/assistant DSNOs were involved in the process of stool collection only $12(60 \%)$ participated in the process for all cases reported in their respective LGAs in 2015 . Additionally, while $9(45 \%)$ of the respondents admit that specimen are batched in Bauchi prior transporting to Gombe, $18(90 \%)$ of the responses shows that specimen are batched in Gombe for onward delivery to the national polio laboratory in Ibadan. Thus, the low NPENT rate (10.3\%) recorded in Bauchi in 2015 could be attributed to factors that include batching of specimens before the final analysis in national polio laboratory, failure of DSNOs/assistant DSNOs to fully participate in the process of stool collection of reported AFP cases. Thus, there is need to avoid batching of stool specimen at all levels to avoid compromising outcome of the final analysis.
\end{abstract}

Keywords: Acute Flaccid Paralysis, AFP Core Indicators, Batching of Specimen, Non-polio Enteroviruses, Non-polio AFP Rate, Reverse Cold Chain

\section{Introduction}

The global fight against poliomyelitis has recorded yet another milestone with the delisting of Nigeria in the last quarter of 2015 from the list of polio endemic countries [1, 3]. Polio, a disease that mainly affects children under the age five years and often associated with poverty, is a highly infectious viral disease transmitted through faeco-oral route. Once a vulnerable unimmunized child is infected, there is a 1 in 200 chance of resulting in irreversible paralysis with a case fatality rate of $5-10 \%$ especially when the respiratory muscles are affected $[1,3]$.
With the World Health Organization at the centre of the global polio programme giving technical support to affected countries, and other key players that include the US Centers for Disease Control and Prevention (CDC), the UNICEF, and other major donors such as the Bill and Melinda Gates Foundation (BMGF), there is a reduction in the number of cases with at least $99 \%[1,4]$.

Hitherto, Nigeria was among the 3 remaining polio endemic countries of the world now leaving only two countries on the list: Afghanistan and Pakistan. It is a significant milestone as numbers of countries that were endemic is down to 2 from more than 125 in 1988. It is reassuring to note that Nigeria is heading towards achieving 
certification as polio free country in 2017 considering the fact that the country in 2015 had a single case of circulating vaccine-derive poliovirus (cVDPV) with no reported wild poliovirus (WPV). This is especially when compared with 2014 when Nigeria had 28 cases of cVDPV and 6 wild polioviruses (WPV). Furthermore, the last reported WPV 1 and WPV 3 in Nigeria had their date of onset of paralysis as $24^{\text {th }}$ July 2014 and $10^{\text {th }}$ November 2012 respectively [1].

Looking at Ghana, a country neighbouring Nigeria, which has cultural similarities, the last case of indigenous wild poliovirus was recorded in 1999; still, the country had two more outbreaks in 2003 and 2008. The result of these two outbreaks made the government double its effort to interrupt the virus through adherence to high routine immunization coverage with liveattenuated oral polio vaccine, improved AFP surveillance and supplementary immunization activities [2].

The sensitivity and specificity of the AFP surveillance is key towards eradicating the disease from across the globe. Thus, the internationally accepted gold standard for the disease detection is through achieving the AFP core indicators. The surveillance system has essentially four steps; these are AFP active case search and reporting of suspected cases, collection and transportation of stool samples for laboratory analysis using the reverse cold chain, laboratory isolation and identification of the poliovirus, and genetic mapping of the virus to determine the origin for classification purposes [1, 4].

Internationally, countries are expected to meet the two AFP core indicators to be able to meet up with the certification process; these indicators are non-polio AFP rate (NP-AFP rate) and the stool adequacy rates $[1,4]$. The NP-AFP rate requires that countries should have at least one detected case of non-polio AFP annually per 100,000 population of children under the age of 15 years $[1,4]$. However, in Nigeria the bar was raised and the requirement is that States should have at least $2 \mathrm{NP}$-AFP rate to meet up with the national standard. Additionally, countries are expected to meet up with the stool adequacy rates of at least $80 \%$ of all specimens collected from suspected AFP cases $[1,4]$.

While two stool specimen are to be sent to the laboratory from every suspected AFP case, the additional requirements are that samples should be at least 8 grams for laboratory analysis, samples must be collected between 24-48 hours apart, samples must be well documented and collected within 14 days of the onset of paralysis, samples must arrive be maintained using the reverse cold chain, and samples must reach the laboratory within 72 hours of collection of the second specimen. Adherence to these guidelines is paramount to ensure that the core indicators are met. Not meeting these requirements would render the specimen inadequate, losing the enteroviruses in the specimen, comprising the outcome of the stool analysis, and leaving the NPENT rate short of the minimum required $\geq 10 \%[1,4]$.

\section{Methods}

This quantitative research study used a closed-ended questionnaire. The questionnaire was applied to a cross section of DSNOs across the 20 LGAs and responses were collated for onward processing. Importantly, the DSNOs were trained by the WHO technical officers at the zonal office. The unit of analysis for the responses was individual, and the measurement instrument was tested for reliability and validity. Using a simple random sampling technique, 20 respondents were selected out of the 24 DSNOs currently on the PEI network in Bauchi State. Using a simple statistical tool, the responses were analysed and inferences drawn accordingly.

The questionnaires deliberately fail to include name of LGA on the questionnaire to protect the identity of respondents. There were two broad columns for demographic information of the respondent and questions centred on when last the DSNO had training on surveillance, cumulative number of AFPs reported in 2015, participation in the process of stool specimen collection and how the specimens were transported to the laboratory for final analysis. The quality of the items on the questionnaire was sought with content validity, and the outcome is valid and generalizable. Thus, the instrument clearly measures what it was expected to measure as validity and reliability of the measurement instrument were well tested $[5,6]$.

\section{Results}

In an earlier study on Bauchi State that looked at the 2015 AFP core indicators, based on results available from the national polio laboratory as at $30^{\text {th }}$ March 2016, it was observed that of the 534 AFPs reported from across the 20 LGAs in the State in 2015, Bauchi and Toro LGAs had highest number of AFP cases each having 50 (9.36\%) [7]. However, in spite the highest number reported by the two LGAs, the NPENT rate was dismal as they fail short of meeting the minimum required NPENT rate of at least $10 \%$.

Analysis of results collated from the questionnaire applied to the 20 DSNOs one from of the LGAs shows that 19 (95\%) males and $1(5 \%)$ female. While all the respondents 20 $(100 \%)$ attended tertiary institutions, $16(80 \%)$ are the substantive DSNOs while 4 (20\%) were assistant DSNOs. Importantly, all the 20 respondents $(100 \%)$ received training in the area of surveillance by the WHO in less than 1 year. Thus, the respondents are up to date on especially AFP surveillance guidelines.

Table 1. Showing collection of stool specimen within 14 days of onset of paralysis.

\begin{tabular}{|lc}
\hline Collection of stool specimen within $\mathbf{1 4}$ days of onset of paralysis \\
\hline Yes & No \\
\hline 20 & 0 \\
\hline
\end{tabular}

Table 1 shows that all the $20(100 \%)$ respondents collected stool samples from all suspected AFP cases within the stipulated 14 days from the date of onset of paralysis, an important requirement in meeting up the stool adequacy and NPENT rate. The other requirements for achieving these two important parameters include obtaining at least 2 grams of 
specimen, avoiding spillage and transporting temperature of +2 to +8 degrees Celsius to the national polio laboratory.

Although all the $20(100 \%)$ respondents participated in the process of at least a sample during the stool collection, Figure 1 shows that there were only $13(65 \%)$ who were fully involved throughout the process of the stool collection. Thus, this could partly explain some of the reasons behind the low NPENT rate recorded in 2015.

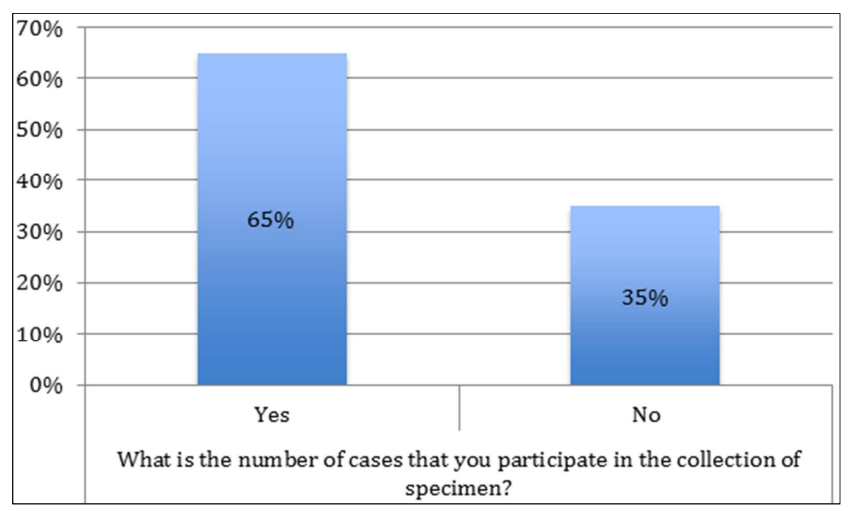

Figure 1. Showing number of reported AFPS DSNOs participated in the collection of stool specimen in 2015.

Table 2. Showing responses on whether DSNOs observes reverse cold chain during transportation of stool specimen.

\begin{tabular}{lc}
\hline $\begin{array}{l}\text { Do you observes reverse cold chain during transportation of stool } \\
\text { specimen to a temperature range of }+2\end{array}$ & to +8 degrees celsius? \\
\hline Yes & No \\
\hline 20 & 0 \\
\hline
\end{tabular}

Table 2 shows that all the $20(100 \%)$ respondents maintain the reverse cold chain practice to ensure that temperature is within +2 to +8 degrees Celsius in the specimen box to the national polio laboratory. The caveat is that failure to maintain the reverse cold chain could result in temperature above +8 degrees Celsius thus affecting viability of the enteroviruses compromising the outcome of the results and eventually leading to low NPENT rate.

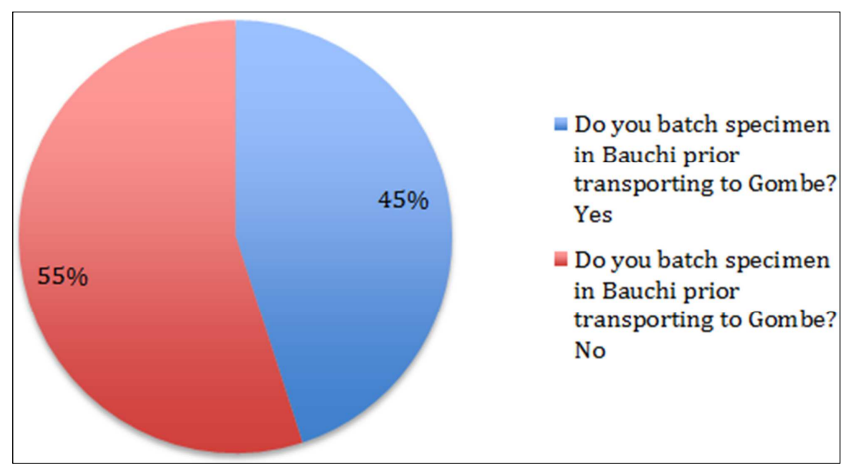

Figure 2. Showing responses on whether specimen are batched in Bauchi prior shipment to Gombe.

The responses as shown in Figure 2 indicates that 9 (45\%) batch their specimen with samples from other LGAs at the central cold store Bauchi, the official designated batching centre at a temperature range of +2 to +8 degrees Celsius, prior transporting to the laboratory in Gombe. However, the batching could equally affect the NPENT rate as some samples are collected from the patients close to day 14 of onset of paralysis thus risking the specimen to become inadequate as well as affect the viability of the enteroviruses.

The responses in Figure 3 indicates that 18 (90\%) stool samples are batched at the Gombe laboratory prior transporting to the national designated polio laboratory in Ibadan for final analysis. Still, batching in Gombe could potentially render some samples inadequate as well as affect viability of the enteroviruses.

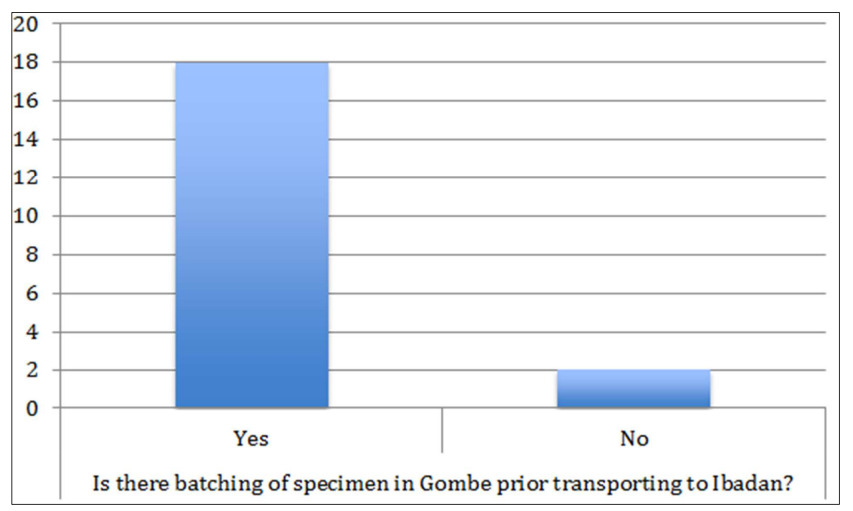

Figure 3. Show responses on whether specimen are batched in Gombe prior shipment to Ibadan.

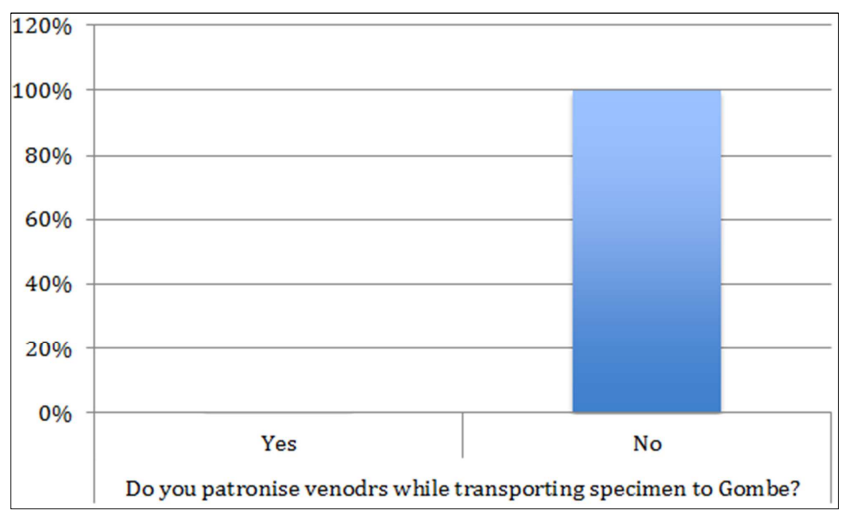

Figure 4. On whether DSNOs patronise vendors while transporting specimen to Gombe.

Additionally, figure 4 shows that all the 20 (100\%) respondents did not patronize vendors in transporting specimen to the transit laboratory in Gombe. The implication of patronizing vendors to transport specimen from Bauchi to Gombe is that the minimum required to transport the samples would not be met adversely affecting the final outcome of the laboratory result and analysis.

\section{Discussions}

In retrospection, it was in early 2014 when insecurity in Borno State northeastern Nigeria resulted in policy change that stool specimen from all suspected AFPs be transported to a laboratory in Gombe for onward transmission to Ibadan national polio laboratory for final analysis. Indeed this policy 
decision has resulted in some unintentional delays in analyzing stool specimen within the stipulated time period to have an NPENT rate that conforms to the standard norms. Thus, the results from this research clearly points to some gaps that could possibly explain the marginal cumulative $10.3 \%$ NPENT rate Bauchi State recorded in 2015. Arguably, looking at the number of AFP cases Bauchi State reported in 2015, the NPENT rate should be far above the minimum required $10 \%$.

In a study involving 4171 stool samples collected from 2097 suspected AFPs in 10 northern States of Nigeria in 2002 and 2003, the result shows that only 307 (14.6\%) cases had NPENTs isolated from their faeces using RD and L20B cell lines. Additionally, and on sixty days follow-up after onset of the 307 cases, only $24(7.8 \%)$ had residual paralysis. Further laboratory analysis revealed that the NPENT cases with residual paralysis are as a result of coxsackie B, echovirus, 3, $4,7,12,13,27,29$ and 33 respectively with $1(4.2 \%)$ mixtures of coxsackie $\mathrm{B}$ and echovirus, 13 (54.2\%) echovirus, $6(25 \%)$ indeterminates and $4(16.7 \%)$ negatives resulting due to loss of virus in storage [8]

Thus, looking at the results from Bauchi, there is need to ensure that the gaps identified in this research are addressed diligently. This is especially considering the fact that Nigeria is approaching 2017, the year designated for the country and the continent as a whole to get certification as polio free. Considering the fact that stools specimen of suspected non-polio AFP cases sent to national polio laboratories should test positive for the enteroviruses to achieve the NPENT rate $[9,10]$ there is need to ensure that the DSNOs and their assistants strictly adhere to the norms of specimen collection as required. Thus, the DSNOs/assistant DSNOs must ensure that they participated fully in the collection of the two specimens from each suspected AFP case. Furthermore, the issue of batching specimen must be looked into and if possible to be avoided to ensure that all specimen collected reaches the final laboratory and get analyzed within the stipulated 14 days from the onset of paralysis; this is paramount as unnecessary delay could destroy the enteroviruses in the specimen, render it inadequate and eventually affect the outcome of the final analysis.

\section{Acknowledgments}

I acknowledge, with gratitude, approval for the use of the AFP dataset from the WHO office Bauchi for the sole purpose of this study.

\section{Conflicts of Interest}

There is no conflict of interest or royalty associated with this study.

\section{References}

[1] WHO (2015). Poliomyelitis. Retrieved from http://www.who.int/mediacentre/factsheets/fs114/en/

[2] Odoom, J. K., Ntim, N. A., Sarkodie, B. et al. (2014) BMC Public Health, 14: 687 Retrieved from http://www.biomedcentral.com/1471-2458/14/687

[3] Global Polio Eradication Initiative (2016). Retrieved from http://www.polioeradication.org/

[4] CDC (2015). The Global Polio Eradication Initiative Stop Transmission of Polio (STOP) Program-1999-2013. Retrieved from

http://www.cdc.gov/mmwr/preview/mmwrhtml/mm6224a5.htm

[5] Frankfort-Nachmias, C., \& Nachmias, D. (2008). Research methods in the social sciences ( $7^{\text {th }}$ ed.). New York: Worth.

[6] Szklo, M., \& Nieto, F. J. (2014). Epidemiology: Beyond the basics $\left(3^{\text {rd }}\right.$ ed.). Sudbury, MA: Jones and Bartlett

[7] Saleh, J-E. A., Vaz, R. G., Braka, F., Abdelrahim, K., \& Ningi, A. I. (2016). A critical look at 2015 AFP surveillance core indicators of Bauchi State, Nigeria. Science Journal of Public Health (In press).

[8] Soji, O. B., Olayinka, O. A., Obu, H. T., et al. (2007). Nonpolio Enteroviruses Implicated in Acute Flaccid Paralysis in Northern Nigeria. Res. J. Medicine \& Med. Sci., 2 (1): 25-28, 2007

[9] CDC (2015). Non-Polio Enterovirus. Retrieved from http://www.cdc.gov/non-polio-enterovirus/

[10] CDC (2013). Non-Polio Enterovirus. Retrieved from http://www.cdc.gov/non-polio-enterovirus/about/ 